\title{
Non-linearity and Non-Gaussianity through Phase Information
}

\author{
Peter Coles ${ }^{1}$ and Lung-Yih Chiang ${ }^{2}$ \\ 1 School of Physics \& Astronomy, University of Nottingham, University Park, \\ Nottingham NG7 2RD, United Kingdom \\ 2 Theoretical Astrophysics Center, Juliane Maries Vej 30, DK-2100 Copenhagen, \\ Denmark
}

\begin{abstract}
In the standard picture of structure formation, initially random-phase fluctuations are amplified by non-linear gravitational instability to produce a final distribution of mass which is highly non-Gaussian and has highly coupled Fourier phases. Second-order statistics, such as the power spectrum, are blind to this kind of phase association. We discuss the information contained in the phases of cosmological density fluctuations and their possible use in statistical analysis tools. In particular, we show how the bispectrum measures a particular form of phase association called quadratic phase coupling, show how to visualise phase association using colour models. These techniques offer the prospect of more complete tests of initial non-Gaussianity than those available at present.
\end{abstract}

\section{Introduction}

The local Universe displays a rich hierarchical pattern of galaxy clusters and superclusters [1]. The early Universe, however, was almost smooth, with only slight ripples seen in the cosmic microwave background radiation [2]. Models of the evolution of structure link these observations through the effect of gravity, because the small initially overdense fluctuations attract additional mass as the Universe expands [3]. During the early stages, the ripples evolve independently, like linear waves on the surface of deep water. As the structures grow in mass, they interact with other in non-linear ways, more like waves breaking in shallow water. Cosmic structure can be characterized by phase correlations associated with these non-linear interactions, but this information is missed by standard analysis techniques such as the power spectrum. In order to do justice to the large data sets about to become available, it is important to design techniques sensitive to the fine details of cosmic structure they will reveal. Here we report a method of quantifying phase information [4] and suggest how this information may be exploited to build novel statistical descriptors that can be used to mine the sky more effectively than with standard methods. 


\section{Fourier Description of Cosmological Density Fields}

In most popular versions of the "gravitational instability" model for the origin of cosmic structure, particularly those involving cosmic inflation [5], the initial fluctuations that seeded the structure formation process form a Gaussian random field [6]. Deviations from uniformity, expressed in terms of the density contrast $\delta(\mathbf{x})$ defined by $\delta(\mathbf{x})=\left[\rho(\mathbf{x})-\rho_{0}\right] / \rho_{0}$, where $\rho_{0}$ is the average density and $\rho(\mathbf{x})$ is the local matter density. Because the initial perturbations evolve linearly, it is useful to expand $\delta(\mathbf{x})$ as a Fourier superposition of plane waves:

$$
\delta(\mathbf{x})=\sum \tilde{\delta}(\mathbf{k}) \exp (i \mathbf{k} \cdot \mathbf{x})
$$

The Fourier transform $\tilde{\delta}(\mathbf{k})$ is complex and therefore possesses both amplitude $|\tilde{\delta}(\mathbf{k})|$ and phase $\phi_{\mathbf{k}}$ where

$$
\tilde{\delta}(\mathbf{k})=|\tilde{\delta}(\mathbf{k})| \exp \left(i \phi_{\mathbf{k}}\right)
$$

Gaussian random fields possess Fourier modes whose real and imaginary parts are independently distributed. In other words, they have phase angles $\phi_{k}$ that are independently distributed and uniformly random on the interval $[0,2 \pi]$. When fluctuations are small, i.e. during the linear regime, the Fourier modes evolve independently and their phases remain random. In the later stages of evolution, however, wave modes begin to couple together [3]. In this regime the phases become non-random and the density field becomes highly non-Gaussian. Phase coupling is therefore a key consequence of nonlinear gravitational processes if the initial conditions are Gaussian and a potentially powerful signature to exploit in statistical tests of this class of models.

A graphic demonstration of the importance of phases in patterns generally is given in Fig 1. Since the amplitude of each Fourier mode is unchanged in the phase reshuffling operation, these two pictures have exactly the same power-spectrum, $P(k) \propto|\tilde{\delta}(\mathbf{k})|^{2}$. In fact, they have more than that: they have exactly the same amplitudes for all $\mathbf{k}$. They also have totally different morphology. The evident shortcomings of $P(k)$ can be partly ameliorated by defining higher-order quantities such as the bispectrum [3, 7, 8,9] or correlations of $\tilde{\delta}(\mathbf{k})^{2}[10$.

\section{The Bispectrum and Phase Coupling}

The bispectrum and higher-order polyspectra vanish for Gaussian fields, but in a non-Gaussian field they may be non-zero. The usefulness of these and related quantities therefore lies in the fact that they encode some information about non-linearity and non-Gaussianity. To understand the relationship between the bispectrum and Fourier phases, it is very helpful to consider the 

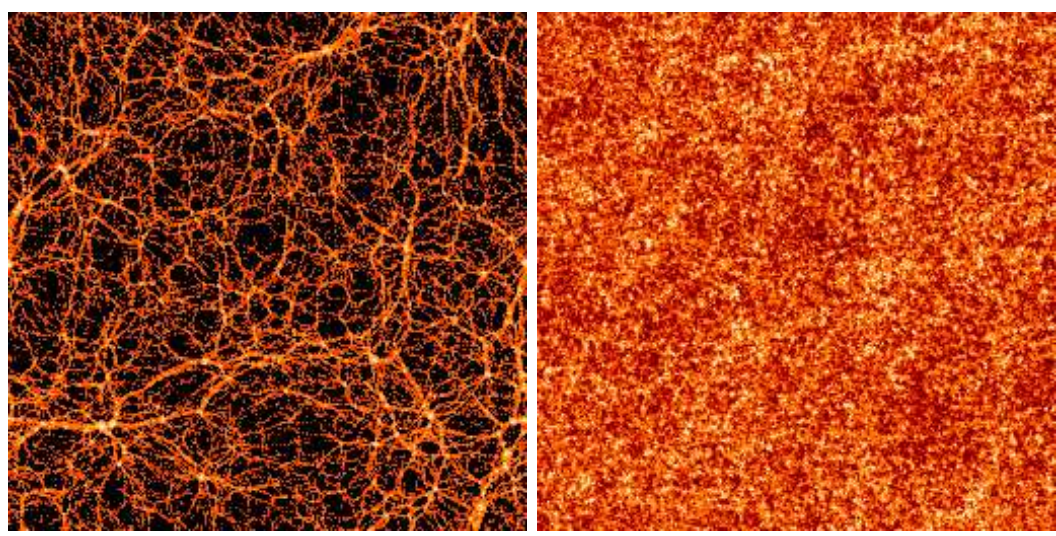

Fig. 1. Numerical simulation of galaxy clustering (left) together with a version generated randomly reshuffling the phases between Fourier modes of the original picture (right).

following toy examples. Imagine a simple density field defined in one spatial dimension that consists of the superposition of two cosine components:

$$
\delta(x)=A_{1} \cos \left(\lambda_{1} x+\phi_{1}\right)+A_{2} \cos \left(\lambda_{2} x+\phi_{2}\right) .
$$

The generalisation to several spatial dimensions is trivial. The phases $\phi_{1}$ and $\phi_{2}$ are random and $A_{1}$ and $A_{2}$ are constants. We can simplify the following by introducing a new notation

$$
\delta(x)=A_{1}\left(\begin{array}{c}
\lambda_{1} \\
\phi_{1}
\end{array}\right)+A_{2}\left(\begin{array}{c}
\lambda_{2} \\
\phi_{2}
\end{array}\right) .
$$

Clearly this example displays no phase correlations. Now consider a new field obtained from the example (3) through the non-linear transformation

$$
\delta(x) \mapsto \delta(x)+\epsilon \delta^{2}(x),
$$

where $\epsilon$ is a constant parameter. Equation (5) may be thought of as a very phenomenological representation of a perturbation series, with $\epsilon$ controlling the level of non-linearity. Using the same notation as equation (值), the new field $\delta(x)$ can be written

$$
\begin{aligned}
\delta(x)= & B_{1}\left(\begin{array}{l}
\lambda_{1} \\
\phi_{1}
\end{array}\right)+B_{2}\left(\begin{array}{l}
\lambda_{2} \\
\phi_{2}
\end{array}\right)+B_{3}\left(\begin{array}{l}
2 \lambda_{1} \\
2 \phi_{1}
\end{array}\right)+B_{4}\left(\begin{array}{l}
2 \lambda_{2} \\
2 \phi_{2}
\end{array}\right)+ \\
& +B_{5}\left(\begin{array}{l}
\lambda_{1}+\lambda_{2} \\
\phi_{1}+\phi_{2}
\end{array}\right)+B_{6}\left(\begin{array}{c}
\lambda_{1}-\lambda_{2} \\
\phi_{1}-\phi_{2}
\end{array}\right)
\end{aligned}
$$

where the $B_{i}$ are constants obtained from the $A_{i}$. Notice in equation (6) that the phases follow the same kind of harmonic relationship as the wavenumbers. This form of phase association is termed quadratic phase coupling. It is 
this form of phase relationship that appears in the bispectrum. To see this, consider another two toy examples. First, model A,

$$
\delta_{\mathrm{A}}(x)=\left(\begin{array}{c}
\lambda_{1} \\
\phi_{1}
\end{array}\right)+\left(\begin{array}{c}
\lambda_{2} \\
\phi_{2}
\end{array}\right)+\left(\begin{array}{c}
\lambda_{3} \\
\phi_{3}
\end{array}\right)
$$

in which $\lambda_{3}=\lambda_{1}+\lambda_{2}$ but in which $\phi_{1}, \phi_{2}$ and $\phi_{3}$ are random; and

$$
\delta_{\mathrm{B}}(x)=\left(\begin{array}{c}
\lambda_{1} \\
\phi_{1}
\end{array}\right)+\left(\begin{array}{c}
\lambda_{2} \\
\phi_{2}
\end{array}\right)+\left(\begin{array}{l}
\lambda_{3}=\lambda_{1}+\lambda_{2} \\
\phi_{3}=\phi_{1}+\phi_{2}
\end{array}\right) .
$$

Model A exhibits no phase association; model B displays quadratic phase coupling. It is straightforward to show that $\left\langle\delta_{A}\right\rangle=\left\langle\delta_{B}\right\rangle=0$. The autocovariances are equal:

$$
\xi_{A}(r)=\left\langle\delta_{A}(x) \delta_{A}(x+r)\right\rangle=\xi_{B}(r)=\frac{1}{2}\left[\cos \left(\lambda_{1} r\right)+\cos \left(\lambda_{2} r\right)+\cos \left(\lambda_{3} r\right)\right],
$$

as are the power spectra, demonstrating that second-order statistics are blind to phase association.

The (reduced) three-point autocovariance function is

$$
\zeta\left(r_{1}, r_{2}\right)=\left\langle\delta(x) \delta\left(x+r_{1}\right) \delta\left(x+r_{2}\right)\right\rangle .
$$

For model A we get

$$
\zeta_{A}\left(r_{1}, r_{2}\right)=0
$$

whereas for model B it is

$$
\begin{aligned}
\zeta_{B}\left(r_{1}, r_{2}\right)= & \frac{1}{4}\left[\cos \left(\lambda_{2} r_{1}+\lambda_{1} r_{2}\right)+\cos \left(\lambda_{3} r_{1}-\lambda_{1} r_{2}\right)+\cos \left(\lambda_{1} r_{1}+\lambda_{2} r_{2}\right)\right. \\
& \left.+\cos \left(\lambda_{3} r_{1}-\lambda_{2} r_{2}\right)+\cos \left(\lambda_{1} r_{1}-\lambda_{3} r_{2}\right)+\cos \left(\lambda_{2} r_{1}-\lambda_{3} r_{2}\right)\right] 12
\end{aligned}
$$

The bispectrum, $B\left(k_{1}, k_{2}\right)$, is defined as the two-dimensional Fourier transform of $\zeta$, so $B_{A}\left(k_{1}, k_{2}\right)=0$ trivially, whereas $B_{B}\left(k_{1}, k_{2}\right)$ consists of a single spike located somewhere in the region of $\left(k_{1}, k_{2}\right)$ space defined by $k_{2} \geq 0$, $k_{1} \geq k_{2}$ and $k_{1}+k_{2} \leq \pi$. If $\lambda_{1} \geq \lambda_{2}$ then the spike appears at $k_{1}=\lambda_{1}$, $k_{2}=\lambda_{2}$ ). Thus the bispectrum measures the phase coupling induced by quadratic nonlinearities. To reinstate the phase information order-by-order requires an infinite hierarchy of polyspectra.

An alternative way of looking at this issue is to note that the information needed to fully specify a non-Gaussian field to arbitrary order (or, in a wider context, the information needed to define an image resides in the complete set of Fourier phases 11. Unfortunately, relatively little is known about the behaviour of Fourier phases in the nonlinear regime of gravitational clustering 12, 13, 14, 15, 16, 17, but it is of great importance to understand phase correlations in order to design efficient statistical tools for the analysis of clustering data. 


\section{Visualizing and Quantifying Phase Information}

A vital first step on the road to a useful quantitative description of phase information is to represent it visually 17]. In colour image display devices, each pixel represents the intensity and colour at that position in the image 18,19. The quantitative specification of colour involves three coordinates describing the location of that pixel in an abstract colour space, designed to reflect as accurately as possible the eye's response to light of different wavelengths. In many devices this colour space is defined in terms of the amount of Red, Green or Blue required to construct the appropriate tone; hence the RGB colour scheme. The scheme we are particularly interested in is based on three different parameters: Hue, Saturation and Brightness. Hue is the term used to distinguish between different basic colours (blue, yellow, red and so on). Saturation refers to the purity of the colour, defined by how much white is mixed with it. A saturated red hue would be a very bright red, whereas a less saturated red would be pink. Brightness indicates the overall intensity of the pixel on a grey scale. The HSB colour model is particularly useful because of the properties of the 'hue' parameter, which is defined as a circular variable. If the Fourier transform of a density map has real part $R$ and imaginary part $I$ then the phase for each wavenumber, given by $\phi=\arctan (I / R)$, can be represented as a hue for that pixel using the colour circle 17 .

The pattern of phase information revealed by this method related to the gravitational dynamics of its origin. For example in our analysis of phase coupling [4] we introduced a quantity $D_{k}$, defined by

$$
D_{k} \equiv \phi_{k+1}-\phi_{k}
$$

which measures the difference in phase of modes with neighbouring wavenumbers in one dimension. We refer to $D_{k}$ as the phase gradient. To apply this idea to a two-dimensional simulation we simply calculate gradients in the $x$ and $y$ directions independently. Since the difference between two circular random variables is itself a circular random variable, the distribution of $D_{k}$ should initially be uniform. As the fluctuations evolve waves begin to collapse, spawning higher-frequency modes in phase with the original [20]. These then interact with other waves to produce a non-uniform distribution of $D_{k}$. For examples, see

http://www. nottingham.ac.uk/ ppzpc/phases/index.html.

It is necessary to develop quantitative measures of phase information that can describe the structure displayed in the colour representations. In the beginning the phases $\phi_{k}$ are random and so are the $D_{k}$ obtained from them. This corresponds to a state of minimal information, or in other words maximum entropy. As information flows into the phases the information content must increase and the entropy decrease. One way to quantify this is by defining an information entropy on the set of phase gradients. One constructs a 
frequency distribution, $f(D)$ of the values of $D_{k}$ obtained from the whole map. The entropy is then defined as

$$
S(D)=-\int f(D) \log [f(D)] d D,
$$

where the integral is taken over all values of $D$, i.e. from 0 to $2 \pi$. The use of $D$, rather than $\phi$ itself, to define entropy is one way of accounting for the lack of translation invariance of $\phi$, a problem that was missed in previous attempts to quantify phase entropy [21]. A uniform distribution of $D$ is a state of maximum entropy (minimum information), corresponding to Gaussian initial conditions (random phases). This maximal value of $S_{\max }=\log (2 \pi)$ is a characteristic of Gaussian fields. As the system evolves it moves into to states of greater information content (i.e. lower entropy). The scaling of $S$ with clustering growth displays interesting properties 络, establishing an important link between the spatial pattern and the physics driving clustering growth.

\section{Discussion}

In fairly recent history, cosmological data sets were sparse and incomplete, and the statistical methods deployed to analyse them were crude. Secondorder statistics, such as $P(k)$ and $\xi(r)$, are blunt instruments that throw away the fine details of the delicate pattern of cosmic structure. These details lie in the distribution of Fourier phases to which second-order statistics are blind. It would not do justice to massively improved data if effort were directed only to better estimates of these quantities. Moreover, as we have shown, phase information provides a unique fingerprint of gravitational instability developed from Gaussian initial conditions (which have maximal phase entropy). Methods such as those we have described above can therefore be used to test this standard paradigm for structure formation. They can also furnish direct tests of the presence of initial non-Gaussianity [22, 23,24]. As the raw material is increasing in both quality and quantity, it is time to refine

our statistical technology so that the subtle and precious artifacts previously ignored can be both detected and extracted.

\section{References}

1. Shectman, S. et al. (1996) The Las Campanas Redshift Survey, Astrophys. J., 470, 172-188

2. Smoot, G.F. et al. (1992) Structure in the COBE Differential Microwave Radiometer First-year Maps, Astrophys. J., 396, L1-L4

3. Peebles, P.J.E. (1980) The Large-scale structure of the Universe. Princeton University Press, Princeton 
4. Chiang, L.-Y., Coles, P. (2000) Phase information and the evolution of cosmological density perturbations, Mon. Not. R. astr. Soc., 311, 809-824

5. Guth, A.H. \& Pi, S.-Y. (1982) Fluctuations in the New Inflationary Universe, Phys. Rev. Lett., 49, 1110-1113

6. Bardeen, J.M., Bond, J.R., Kaiser, N., Szalay A.S. (1986) The statistics of peaks of Gaussian Random Fields, Astrophys. J., 304, 15-61

7. Matarrese, S., Verde, L., Heavens, A.F. (1997) Large-scale Bias in the Universe: Bispectrum Method, Mon. Not. R. astr. Soc., 290, 651-662

8. Scoccimarro, R., Couchman, H.M.P., Frieman, J.A. (1999) The bispectrum as a signature of gravitational instability in redshift space, Astrophys. J., 517, 531-540 (1999)

9. Verde, L., Wang, L., Heavens, A.F., Kamionkowski, M. (2000) Large-scale Structure, the cosmic microwave background, and primordial non-Gaussianity, Mon. Not. R. astr. Soc., 313, 141-147 (2000)

10. Stirling, A.J., Peacock, J.A. (1996) Power correlations in cosmology: Limits on primordial non- Gaussian density fields, Mon. Not. R. astr. Soc., 283, L99-L104 (1996)

11. Oppenheim, A.V., Lim, J.S. (1981) The Importance of Phase in Signals, Proc. IEEE., 69, 529-541

12. Ryden, B.S., Gramann, M. (1991) Phase Shifts in Gravitationally Evolving Density Fields, Astrophys. J., 383, L33-L36

13. Scherrer, R.J., Melott, A.L., \& Shandarin, S. F. (1991) A Quantitative Measure of Phase Correlations in Density Fields, Astrophys. J., 377, 29-35

14. Soda, J., Suto, Y. (1992) Nonlinear Gravitational Evolution of Phases and Amplitudes in One-dimensional Cosmological Density Fields, Astrophys. J., 396, 379-394

15. Jain, B., Bertschinger, E. (1996) Self-similar evolution of gravitational clustering: is $n=1$ special?, Astrophys. J., 456, 43-54

16. Jain, B., Bertschinger, E. (1998) Self-similar evolution of gravitational clustering: N-body simulations of the $n=-2$ spectrum, Astrophys. J., 509, 517-530

17. Coles, P., Chiang, L.-Y. (2000) Characterizing the non-linear growth of largescale structure in the Universe, Nature, 406, 376-378.

18. Thornton, A.L. (1998) Colour object recognition using a complex colour representation and the frequency domain. PhD Thesis, University of Reading

19. Foley, J.D., Van Dam, A. (1982) Fundamentals of Interactive Computer Graphics (Addison-Wesley, Reading, Mass.

20. Shandarin, S.F., Zel'dovich, Ya. B. (1989) The large-scale structure: turbulence, intermittency, structures in a self-gravitating medium, Rev. Mod. Phys., 61, 185220

21. Polygiannikis, J.M., Moussas, X. (1995) Detection of nonlinear dynamics in solar wind and a comet using phase-correlation measures, Solar Physics, 158, 159-172

22. Ferreira, P.G., Magueijo, J., Górski, K.M. (1998) Evidence for non-Gaussianity in the COBE DMR 4-year sky maps, Astrophys. J., 503, L1-L4

23. Pando, J., Valls-Gabaud, D., Fang, L.-Z. (1998) Evidence for scale-scale correlations in the cosmic microwave background radiation, Phys. Rev. Lett., 81, 4568-4571

24. Bromley, B.C., Tegmark, M. (1999) Is the microwave background really nonGaussian?, Astrophys. J., 524, L79-82 